\title{
Transient complete right bundle branch block following right heart catheterization in children - a report of two cases
}

\author{
Tomasz M. Książczyk, Agnieszka Tomik, Krzysztof Godlewski, Bożena Werner \\ Department of Pediatric Cardiology and General Pediatrics, Medical University of Warsaw, Warsaw, Poland
}

Adv Interv Cardiol 2016; 12, 4 (46): 372-376 DOI: $10.5114 /$ aic.2016.63640

\section{Introduction}

Right bundle branch block (RBBB) in children is usually acquired following cardiac operations. Other causes include congenital heart defects (e.g. primum atrial septal defect (ASD), Ebstein's anomaly) [1-3]. It can be a permanent disorder, usually after cardiac procedures, or it may have a transient character (tRBBB). Transient right bundle branch block is very rare in children and can occur for example as a response to blunt chest trauma [4].

We report 2 cases of complete transient RBBB that were associated with right heart catheterizations, one after pulmonary balloon valvuloplasty and the second one during the Rashkind procedure.

\section{Case reports}

Case 1

A 6-month-old boy with pulmonary valve stenosis (PS) was treated with balloon valvuloplasty. The treatment managed to decrease the invasive peak systolic pressure gradient between the right ventricle (RV) and pulmonary artery (PA) from $55 \mathrm{~mm} \mathrm{Hg}$ to $16 \mathrm{~mm} \mathrm{Hg}$. No complications were observed during or after the procedure. Routine ECG performed before the treatment showed normal sinus rhythm, narrow QRS complexes, and right heart axis. Follow up 12-lead ECG performed $24 \mathrm{~h}$ after the procedure showed sinus rhythm, 125/ min, the QRS prolongation to $100 \mathrm{~ms}$, with typical RBBB morphology, allowing us to diagnose complete RBBB (Figures 1 A, B). 24-hour Holter ECG monitoring was performed, showing sinus rhythm, average HR 130/min and no arrhythmia. The patient was in an excellent clinical condition, showing no signs of distress or heart failure, with a heart rate of $100-150 / \mathrm{min}$. No treatment was administered. In the 12-lead ECG performed $72 \mathrm{~h}$ after the procedure (Figures $1 C, D$ ) the RBBB had resolved. At 3-month follow-up there was no recurrence of the RBBB in the ECG.

\section{Case 2}

A 4-day-old male newborn diagnosed with a complex transposition of the great arteries (d-TGA), ventricular septal defect (VSD) and pulmonary artery stenosis underwent the Rashkind procedure due to the restrictive flow through the interatrial septum. The patient was in a severe general condition before the procedure, with blood oxygenation around $70-75 \%$ and significant dyspnea. His ECG showed normal sinus rhythm and narrow QRS complexes. At the beginning of the procedure during the attempt to forward the catheter through the ASD it was unintentionally placed in the right ventricle, and after that there was an abrupt change in the ECG pattern. We observed widening of the QRS complexes, and there was no change in hemodynamic parameters. Follow-up ECG showed RBBB morphology and QRS prolongation to 100 ms (Figures $2 \mathrm{~A}, \mathrm{~B})$. The procedure was successful; ASD was widened to $8 \times 5 \mathrm{~mm}(2.5 \mathrm{~mm}$ before the procedure), with unrestricted bidirectional flow. After the procedure there was improvement in the patient's general condition and an increase in blood oxygenation to $85 \%$. In the consecutive ECG examinations there was no change; there was still RBBB morphology with $100 \mathrm{~ms}$ QRS duration. On the fifth day after the procedure, because of the persistence of the RBBB we decided to start treatment with i.v. steroids (dexamethasone $0.2 \mathrm{mg} / \mathrm{kg}$ ). On the second day of treatment ECG showed complete resolution of conduction abnormalities (Figures $2 \mathrm{C}$, D). No conduction abnormalities were observed during 6 months of follow-up.

\section{Corresponding author:}

Prof. Bożena Werner MD, PhD, Department of Pediatric Cardiology and General Pediatrics, Medical University of Warsaw, 63 A Żwirki i Wigury St, 02-091 Warsaw, Poland, phone: +48 2231795 75, e-mail: bozena.werner@wum.edu.pl

Accepted: 19.01.2016, accepted: 2.06.2016. 
A

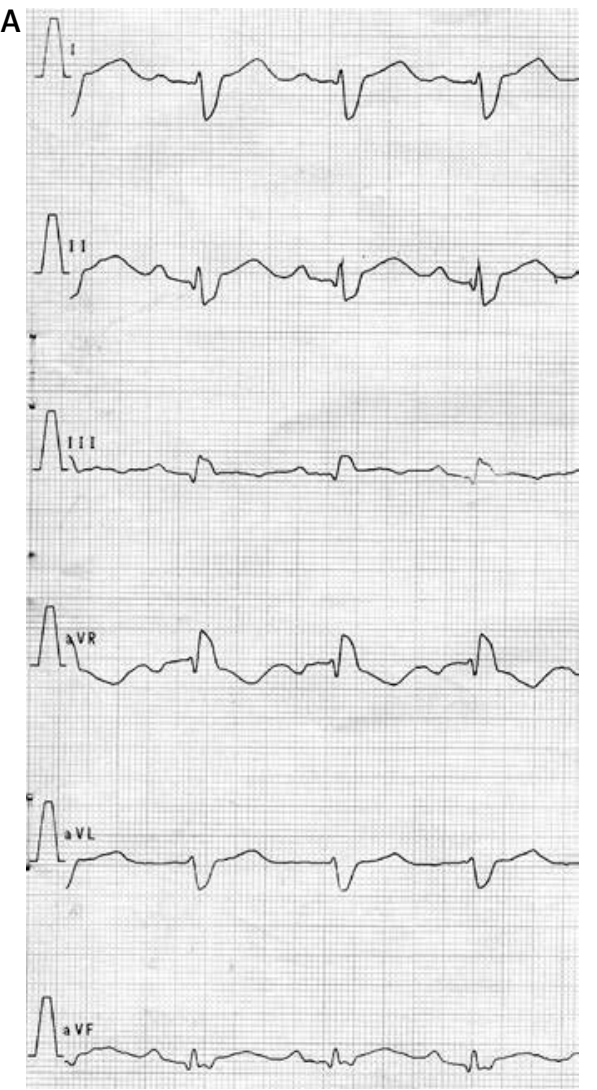

C

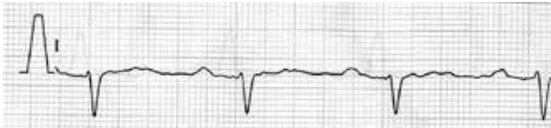

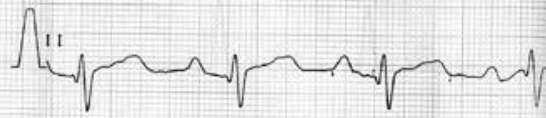
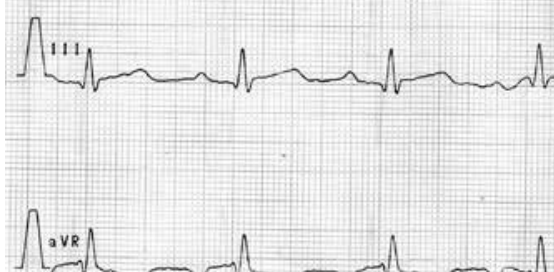

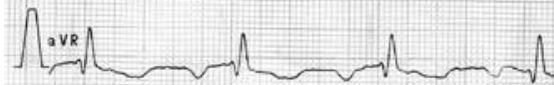

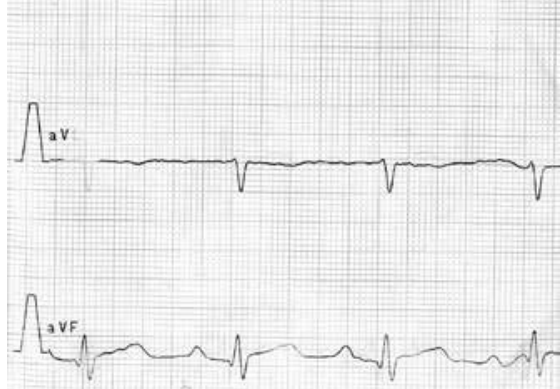

B

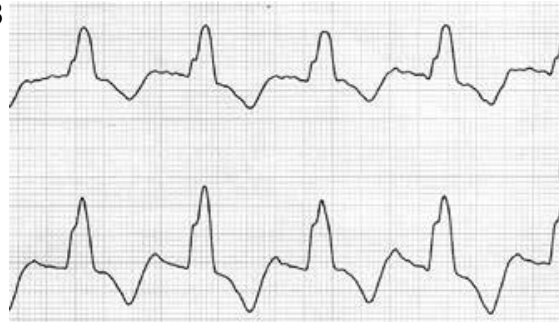

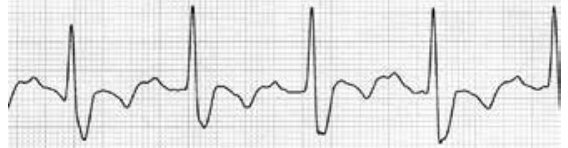

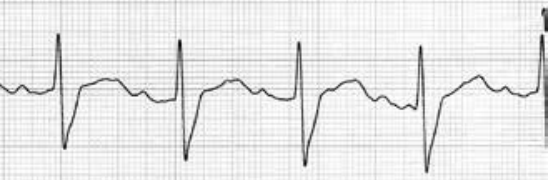

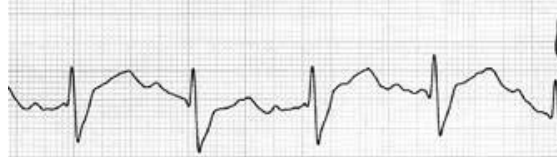

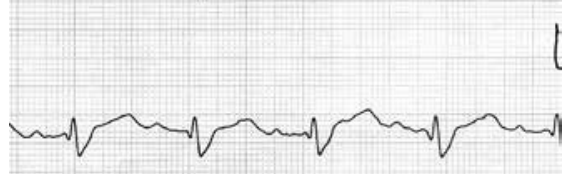

D
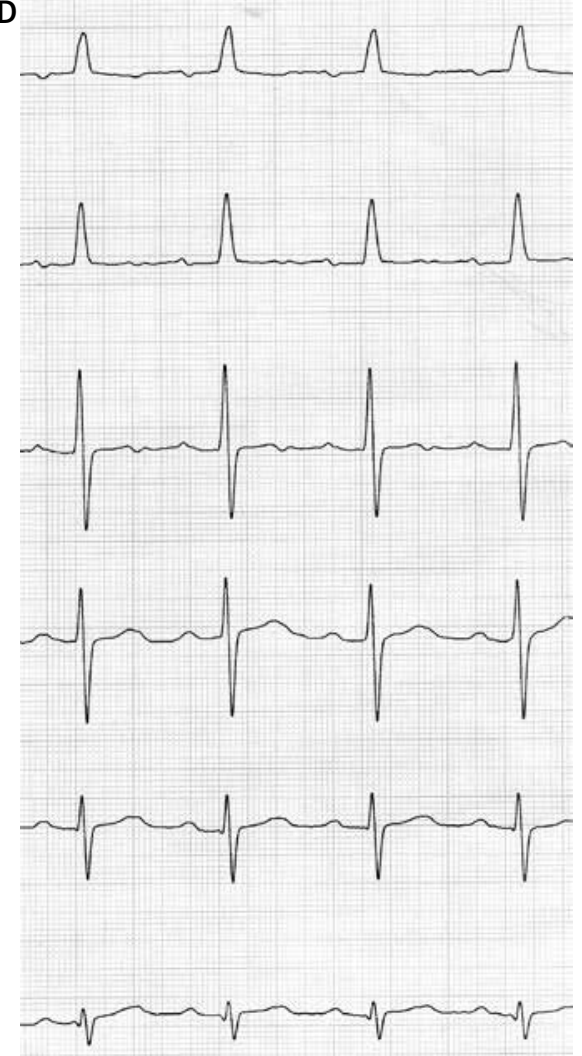

Figure 1. A - sinus rhythm 120/min, PQ 0.14 s, QRS 0.12 s, complete right bundle branch block pattern, B - sinus rhythm 120/min, PQ $0.14 \mathrm{~s}$, QRS $0.12 \mathrm{~s}$, complete right bundle branch block pattern, C - sinus rhythm 110/ min, PQ 0.14 s, QRS 0.06 s, D - sinus rhythm 110/min, PQ 0.14 s, QRS 0.06 s 
A

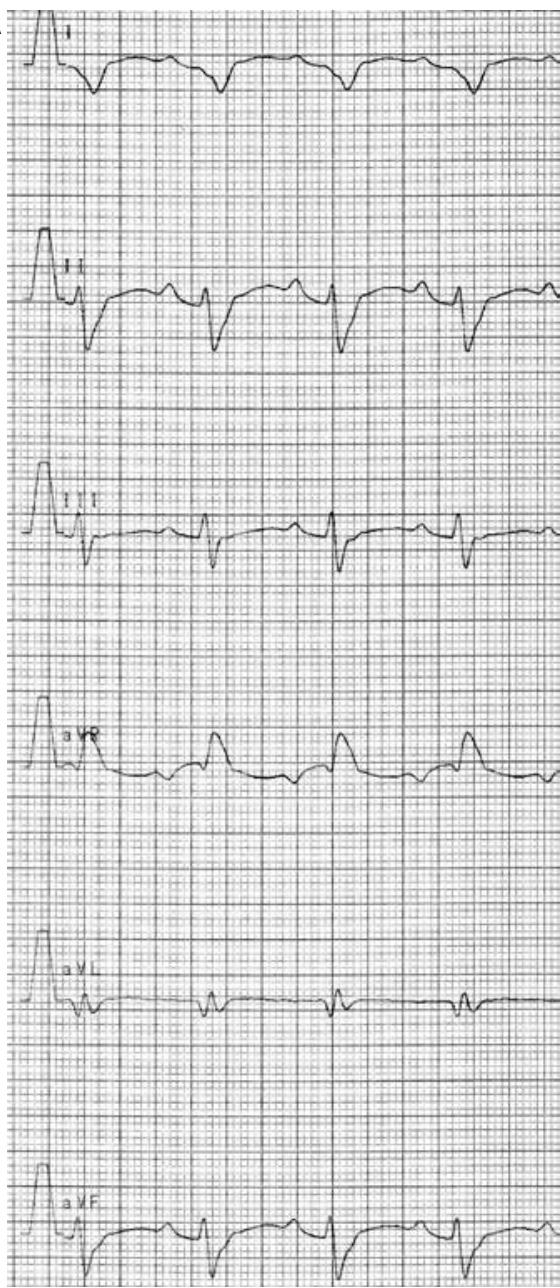

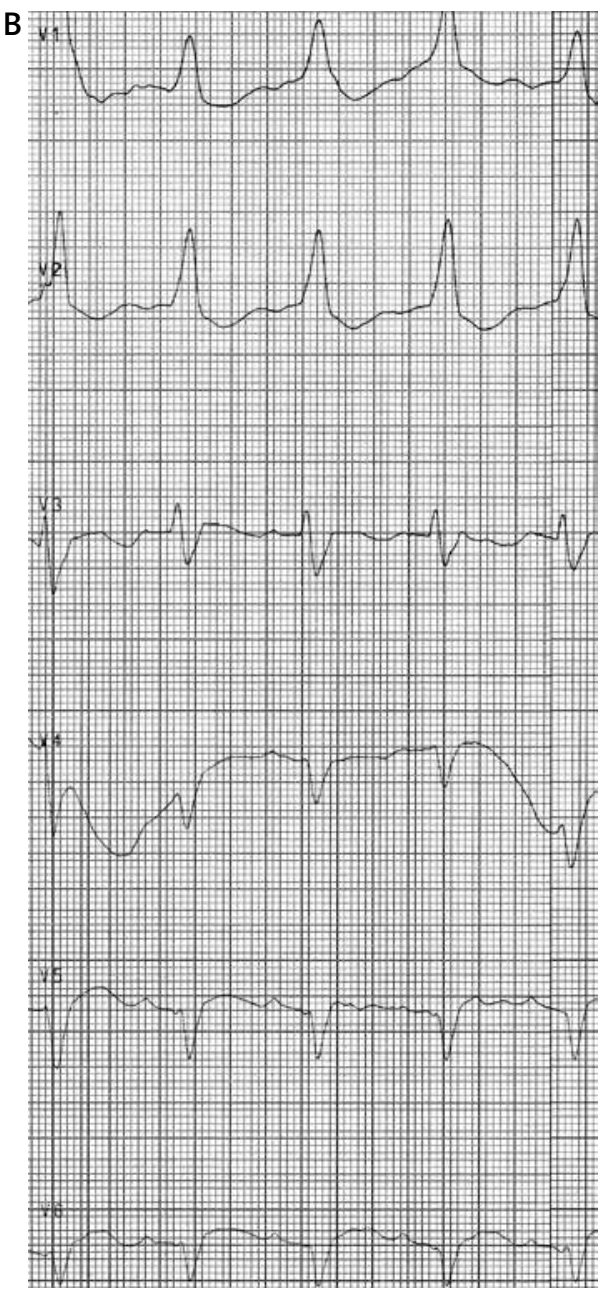

Figure 2. A - sinus rhythm around 160/min, PQ $0.1 \mathrm{~s}$, QRS $0.1 \mathrm{~s}$, right bundle branch block pattern, B - sinus rhythm around 160/min, PQ $0.1 \mathrm{~s}$, QRS $0.1 \mathrm{~s}$, right bundle branch block pattern

\section{Discussion}

Data on prevalence of RBBB in children are limited. Japanese investigators performed a study on a pediatric population comprising over 20,000 children, and complete RBBB was found in $0.16 \%$ and incomplete RBBB in $2.94 \%$ of children [5]. Transient blocks are rarely reported in children. There are reports of transient RBBB following cardiac catheterization for electrophysiological studies $[6,7]$ and both transient RBBB and complete heart block, after a blunt chest trauma [8,9] - the latter one showing that damage to the conduction tract can be caused not only by a direct trauma and can be associated with edema of the conductive tissue. The conduction disturbances discussed in the literature lasted from several minutes to $24 \mathrm{~h}[4,7]$. Some cases of transient RBBB may remain undiagnosed, due to the fact that they may resolve before any follow-up ECG is done.

There are few reports of transient RBBB and complete heart blocks that have occurred after right heart catheterization or central venous catheter placement in adult patients [10]. The incidence of RBBB during right heart catheterization in adults is estimated at 3-12\% [10]. There are no case reports of transient RBBB either during or after interventional cardiology procedures in children.

In the first presented case as a reason of the occurrence of transient RBBB we considered distention of the right bundle branch by the balloon during valvuloplasty and/or distention of the whole right ventricle caused by the temporary obstruction of the right ventricle outflow tract. This mechanism could cause the protraction of the conduction tract and result in prolongation of time needed for the impulse to be transmitted. As for the second case, we wonder if the direct injury to the bundle was responsible for the transient block, and the empirical treatment with iv steroids was successful because it limits edema and trauma done to the conduction tract and its surroundings. In both described cases the edema of the tissues surrounding the right bundle branch, as a result of the trauma caused by the balloon or the catheter, during right heart catheterization could play a role, caus- 

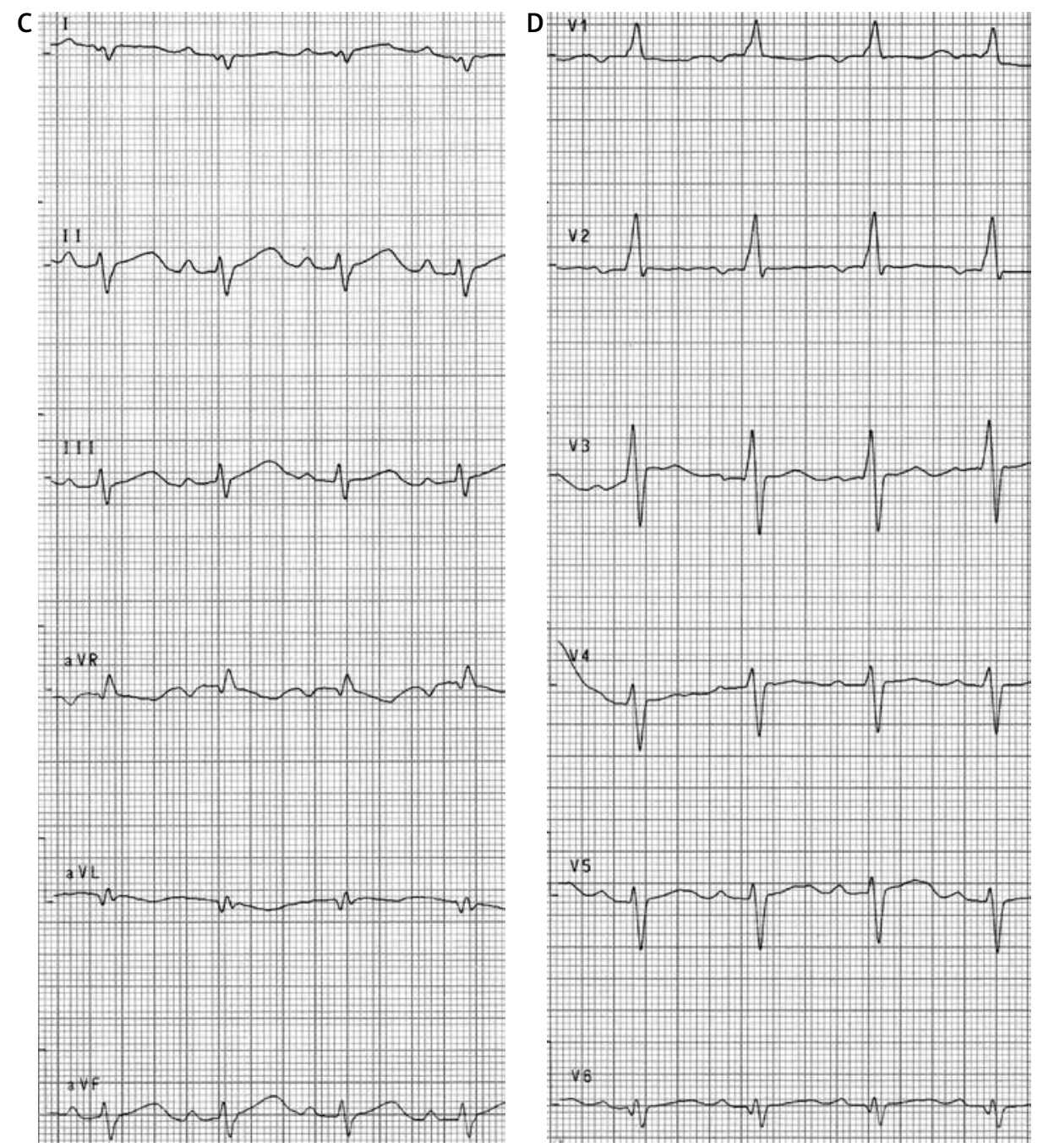

Figure 2. C - sinus rhythm around 160/min, PQ 0.1 s, QRS 0.06 s, D - sinus rhythm around 160/min, PQ 0.1 s, QRS $0.06 \mathrm{~s}$

ing slowdown in the conductance of the impulse through the conduction tract. In the literature there are reports of transient complete heart blocks in children which occurred following interventional procedures such as percutaneous pulmonary valve implantation or closure of the ventricular septal defect $[11,12]$. The recovery time, from complete heart block to sinus rhythm, in the mentioned cases was 3 weeks to 24 months, and in 1 case the authors decided to use steroid treatment for complete heart block. Some authors suggest an empirical bolus of steroids for complete heart block occurring during cardiac interventional procedures, in order to minimize trauma and edema in the tissues surrounding the bundle $[9,12]$.

Transient RBBB is usually a benign disorder, but it can be fatal for a patient with preexisting LBBB, because it can lead to a complete heart block and serious hemodynamic consequences $[4,10,13]$.

In the presented cases we show that the possible injury of the conduction tract has to be always kept in mind when performing cardiac or central vein catheterization.
Although transient RBBB is a benign conduction disorder, and can resolve spontaneously within minutes to days, it can cause serious sequelae if occurring in a patient with preexisting conduction disturbances. In case of hemodynamic disturbances or persistence of the RBBB one can consider administration of iv steroids.

\section{Conflict of interest}

The authors declare no conflict of interest.

\section{References}

1. Okoromo EO, Guller B, Maloney JD, et al. Etiology of right bundle-branch block pattern after surgical closure of ventricular septal defects. Am Heart J 1975; 90: 14-8.

2. Lev M, Unger PN, Lesser ME, et al. Pathology of the conduction system in acquired heart disease: complete right bundle branch block. Am Heart J 1961; 61: 593-9.

3. Horowitz LN, Alexander JA, Edmunds LH. Postoperative right bundle branch block: identification of three levels of block. Circulation 1980; 62: 319-28. 
4. Unnikrishnan D, Idris N, Varshneya N. Complete heart block during central venous catheter placement in a patient with pre-existing left bundle branch block. Br J Anaest 2003; 91: 747-9.

5. Yamakawa Y, Ishikawa T, Uchino K, et al. Prevalence of right bundle-branch block and right precordial ST-segment elevation (Brugada-type electrocardiogram) in Japanese children. Circ 2004; 68: 275-9.

6. Ramos A, Wolff GS, Gelband H. Site of conduction delay in children with catheter-induced right bundle branch block. J Electrocardiol 1983; 16: 41-4

7. Gatzoulis MA, Till JA, Somerville J, et al. Mechano-electrical interaction in tetralogy of Fallot: QRS prolongation relates to right ventricular size and predicts malignant ventricular arrhythmias and sudden death. Circulation 1995; 92: 231-7.

8. Udink ten Cate F, van Heerde M, Rammeloo LA, Hruda J. Transient electrocardiographic abnormalities following blunt chest trauma in a child. Eur J Pediatr 2008; 167: 1331-3.

9. Fujita S, Nakamura T, Koura U, et al. Commotio Cordis presenting as a temporary complete atrioventricular block in a 2-year-old girl with congenitally corrected transposition of the great arteries. Pediatr Cardiol 2011; 32: 1043-7.

10. Morris D, Mulvihill D, Lew WY. Risk of developing complete heart block during bedside pulmonary artery catheterization in patients with left bundle-branch block. Arch Intern Med 1987; 147: 2005-10.

11. Ruf B, Eicken A, Hess J. Transient complete atrioventricular block after percutaneous pulmonary valve implantation. Cardiol Young 2010; 20: 704-6.

12. Erdem S, Kiziltas A, Kucukosmanoglu O, Ozbarlas N. Temporary atrioventricular complete block that develops following the transcatheter closure of ventricular septal defect. Turk J Pediatr 2012; 54: 80-2.

13. Sprung CL, Elser B, Schein RM, et al. Risk of right bundle-branch block and complete heart block during pulmonary artery catheterization. Crit Care Med 1989; 17: 1-3. 